\title{
KEYNOTE-826: A Phase 3, Randomized, Double-blind, Placebo- Controlled Study of Pembrolizumab Plus Chemotherapy for First-line Treatment of Persistent, Recurrent, or Metastatic Cervical Cancer
}

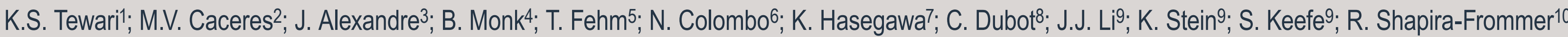
University of California, Irvine Medical Center, Orange, CA, USA; 2 Instituto Ángel H Roffo, Buenos Aires, Argentina; ${ }^{3 H o ̂ p i t a l ~ C o c h i n, ~ P a r i s, ~ F r a n c e ; ~}{ }^{4}$ University of Arizona Cancer Center, Tucson, AZ, USA; 5Heinrich-Heine-Universität Düsseldorf, Düsseldorf, Germany; 6 University of Milan-Bicocca, Milan, Italy; ${ }^{7}$ Saitama Medical University International, Saitama Prefecture, Japan; 8 Institut Curie, Paris, France;

${ }^{9}$ Merck \& Co., Inc., Kenilworth, NJ, USA; ${ }^{10 S h e b a ~ M e d i c a l ~ C e n t e r, ~ R a m a t ~ G a n, ~ I s r a e l ~}$

\section{BACKGROUND}

- Cervical cancer arises in the setting of persistent infection with high-risk human papillomavirus subtypes

-Women with recurrent/metastatic disease represent a poor prognostic group with unmet clinical needs

- Incorporation of antiangiogenesis therapy to standard treatment has emerged as a therapeutic option, but the survival benefit of 3.7 months over chemotherapy alone is modest ${ }^{1}$

- Pembrolizumab is a selective, humanized immunoglobulin G4K monoclonal antibody that prevents the interaction between programmed death receptor-1 (PD-1), expressed by lymphocytes, and its ligands, PD-L1 and PD-L2, expressed by tumor cells (Figure 1) ${ }^{2}$

- On the basis of the $14.3 \%$ objective response for pembrolizumab monotherapy in the cervical cancer cohort of KEYNOTE-158, 3 the US Food and Drug Administration granted accelerated approval to pembrolizumab in June 2018 for treatment of patients with recurrent or metastatic cervical cancer with disease progression on or after chemotherapy whose tumors express PD-L1 (combined positive score $\geq 1)^{2}$

On the basis of these promising results and the unmet clinical needs, combination therapy with pembrolizumab and chemotherapy will be investigated in a first-line setting for advanced cervical cancer

Figure 1. Pembrolizumab and the PD-1 Pathway

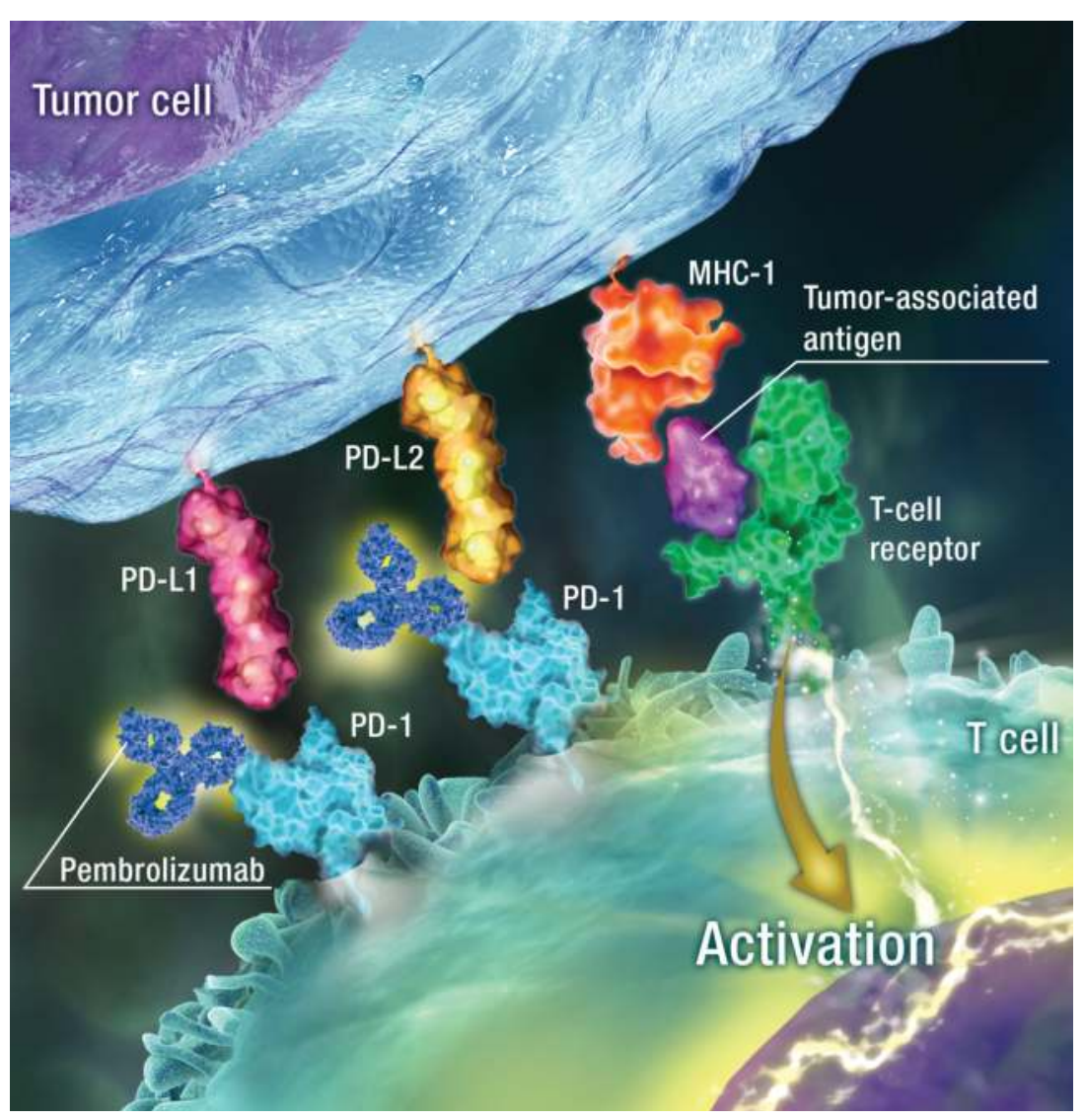

MHC-1, major histocompatibility complex 1; PD-1, programmed death receptor-1; PD-L1, programmed death ligand-1; PD-L2, programmed death ligand-2.

\section{OBJECTIVES}

\section{Primary}

- Evaluate overall survival (OS) and progressionfree survival (PFS), as assessed by blinded independent radiology review via RECIST v1.1, of frontline chemotherapy with and without pembrolizumab in women with recurrent and/or metastatic cervical cancer

\section{Secondary}

- Evaluate objective response, duration of response, 12-month PFS, health-related quality of life, and safety and tolerability

\section{DESIGN}

- KEYNOTE-826 is a phase 3, randomized double-blind, placebo-controlled, multinational trial of pembrolizumab + chemotherapy vs placebo + chemotherapy for first-line treatment of recurrent, persistent, or metastatic cervical cancer (ClinicalTrials.gov identifier, NCT03635567)

Eligible patients will be randomized 1:1 to pembrolizumab + chemotherapy $200 \mathrm{mg}$ intravenously (IV) or placebo + chemotherapy every 3 weeks (Figure 2)

The chemotherapy regimen (paclitaxe $175 \mathrm{mg} / \mathrm{m}^{2} \mathrm{IV}+$ cisplatin $50 \mathrm{mg} / \mathrm{m}^{2}$ or carboplatin AUC $5 \mathrm{IV}$, with or without bevacizumab $15 \mathrm{mg} / \mathrm{kg}$ IV) will be selected by the investigator before randomization

- Patients will be stratified according to metastatic status at diagnosis (primary metastatic or recurrent disease), planned bevacizumab use (yes or no), and PD-L1 status (combined positive score $<1,1$ to $<10$, or $\geq 10$ )

Figure 2. KEYNOTE-826 Study Design
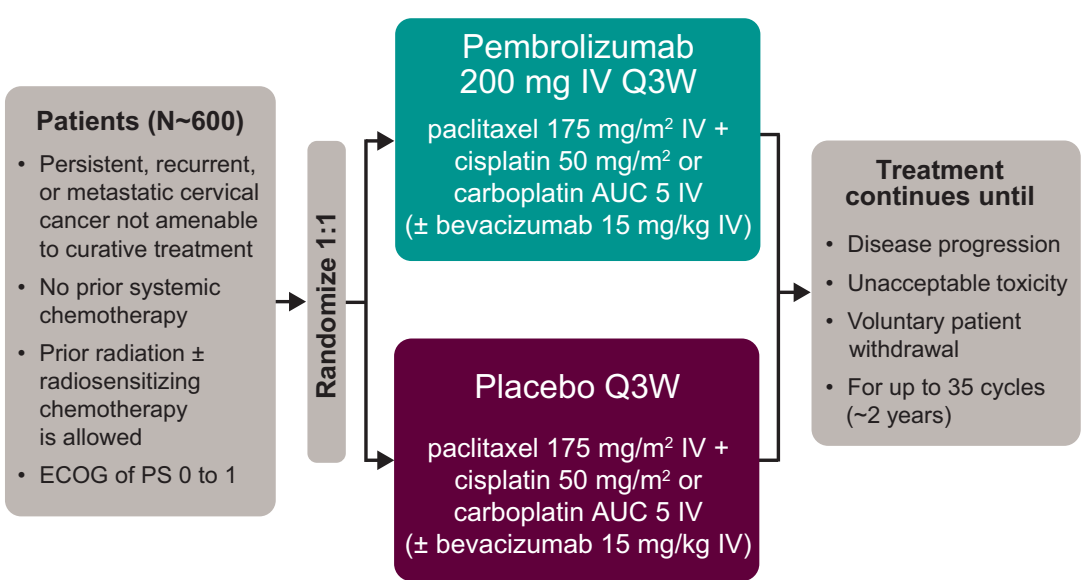

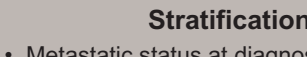

\section{- Prannedic status at diagnosis (yes}

- PD-L1 status (CPS $<1,1$ to $<10$, or $\geq 10$ )

Study treatments administered on day 1 of each 3-week treatment cycle; cisplatin may be administered on day 2 of each cycle per local practice. CPS, combined positive score; ECOG, Eastern Cooperative Oncology Group; IV, intravenous; PD-L1, programmed death ligand-1; PS, performance status; Q3W, every 3 weeks.

\section{Endpoints and Assessments}

\section{- Primary endpoints}

PFS per RECIST v1.1 (assessed by blinded independent central review)

OS

- Secondary endpoints

Objective response

Duration of response

12-month PFS

Patient-reported quality of life using the European Organisation for Research and Treatment of Cancer global score (questionnaires administered before dosing on day 1 of cycles $1-14$, every other cycle thereafter, at end of treatment, and at safety follow-up)

Safety and tolerability

- Imaging assessments of disease status will be performed every 9 weeks from the date of randomization through week 54 and every 12 weeks thereafter

\section{Patient Eligibility Criteria}

\begin{tabular}{|c|}
\hline Key Inclusion Criteria \\
\hline - Women aged $\geq 18$ years \\
\hline $\begin{array}{l}\text { - Persistent, recurrent, or metastatic squamous } \\
\text { cell carcinoma, adenosquamous carcinoma, } \\
\text { or adenocarcinoma of the cervix not previously } \\
\text { treated with systemic chemotherapy and not } \\
\text { amenable to curative treatment (eg, surgery } \\
\text { or radiation)a }\end{array}$ \\
\hline - Measurable disease per RECIST v1.1 criteria \\
\hline - ECOG PS of 0 to 1 \\
\hline Key Exclusion Criteria \\
\hline $\begin{array}{l}\text { - Pregnant or positive urine pregnancy test within } \\
72 \text { hours before randomization, breastfeeding, } \\
\text { or of childbearing potential and not using } \\
\text { contraception during treatment period }\end{array}$ \\
\hline $\begin{array}{l}\text { - Active central nervous system metastases, } \\
\text { carcinomatous meningitis, or additional } \\
\text { malignancy that is progressing or has required } \\
\text { active treatment }\end{array}$ \\
\hline $\begin{array}{l}\text { - Diagnosis of immunodeficiency or receiving } \\
\text { immunosuppressive therapy }\end{array}$ \\
\hline - Active infection requiring systemic therapy \\
\hline $\begin{array}{l}\text { - Prior therapy with an anti-PD-1, anti-PD-L1, } \\
\text { or anti-PD-L2 agent or with an agent directed } \\
\text { at another stimulatory or co-inhibitory } \\
\text { T-cell receptor }\end{array}$ \\
\hline $\begin{array}{l}\text { ECOG, Eastern Cooperative Oncology Group; PD-1, programmed } \\
\text { death receptor-1; PD-L1, programmed death ligand-1; } 1 \text {, } \\
\text { PD-L2, programmed death ligand }-2 \text {; PS, performance status. } \\
\text { aPrior chemotherapy utilized as a radiosensitizing agent and completed } \\
\geq 2 \text { weeks before randomization with resolution of all radiation-related } \\
\text { toxicities is allowed. }\end{array}$ \\
\hline STATUS \\
\hline
\end{tabular}

- Planned enrollment is approximately 600 patients from 126 sites in the United States, Argentina, Australia, Canada, Chile, Colombia, France, Germany, Israel, Italy, Japan, Republic of Korea, Mexico, Peru, Russia, Spain, Taiwan, Turkey, and Ukraine (Figure 3)

Figure 3. Countries with Planned Enrollment Sites for KEYNOTE-826 (shown in green)

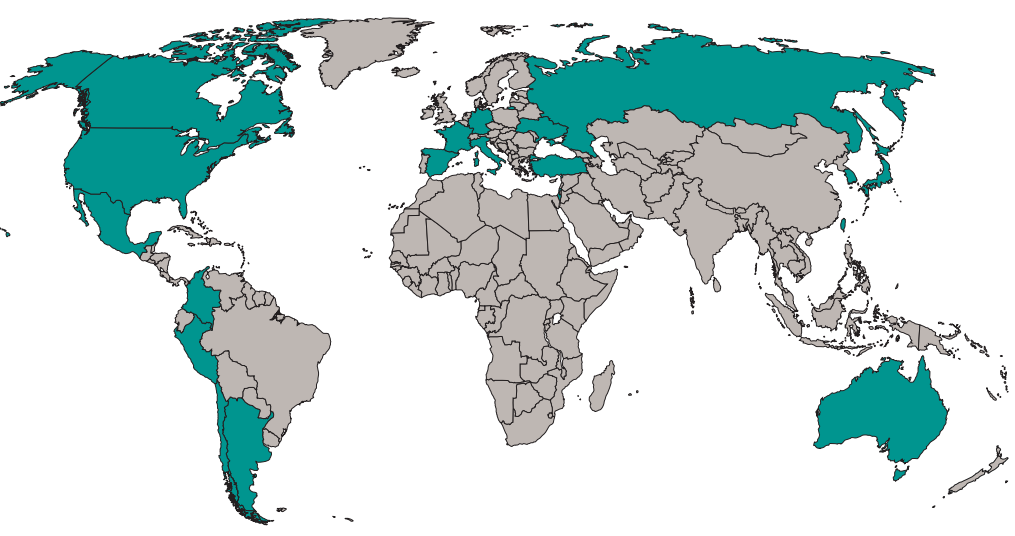

References

1. Tewari KS et al. N Engl J Med. 2014;370:734-743.

. Merck Sharp \& Dohme Corp., KEYTRUDA® (pembrolizumab) injection, for intravenous use. Whitehouse Station, NJ USA; 09/2017. 3. Chung HC et al. J Clin Oncol. 2019 [Epub ahead of print].

\section{Acknowledgments}

Funding for this study was provided by Merck Sharp \& Dohme Corp., a subsidiary of Merck \& Co., Inc., Kenilworth, NJ, USA. Medical writing and editorial assistance were provided by Nathan Rodeberg, PhD, and Jenna Sharp \& Dohme Corp., a subsidiary of Merck \& Co. Inc.. Kenilworth, NJ, USA. Data included in this poster have been presented in full at the ASCO Annual Meeting; May 31-June 4, 2019; Chicago, IL, USA; abstract TPS5603 and at the 2019 JSMO Annual Meeting; July 18-20, 2019;

Contact Information

Contact Krishnansu S. Tewari at ktewari@uciedu
for questions or comments. 